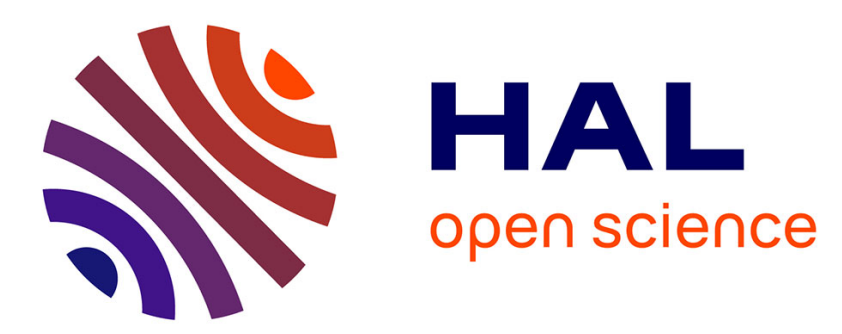

\title{
Aspect zéro et dépendance situationnelle: l'exemple du wolof
}

\author{
Stéphane Robert
}

\section{To cite this version:}

Stéphane Robert. Aspect zéro et dépendance situationnelle: l'exemple du wolof. Claude Müller. Dépendance et intégration syntaxique (subordination, coordination, connexion, Niemeyer, pp.153-161, 1996, Linguistische Arbeiten $n^{\circ}$ 351. hal-00382677

\section{HAL Id: hal-00382677 https://hal.science/hal-00382677}

Submitted on 11 May 2009

HAL is a multi-disciplinary open access archive for the deposit and dissemination of scientific research documents, whether they are published or not. The documents may come from teaching and research institutions in France or abroad, or from public or private research centers.
L'archive ouverte pluridisciplinaire HAL, est destinée au dépôt et à la diffusion de documents scientifiques de niveau recherche, publiés ou non, émanant des établissements d'enseignement et de recherche français ou étrangers, des laboratoires publics ou privés. 
Claude Muller (éd.)

\section{Dépendance et intégration syntaxique; subordination, coordination, connexion}

Actes du colloque "Dépendance et intégration syntaxique"

Université Michel de Montaigne Bordeaux

(5-8 octobre 1994)

1996

Linguistische Arbeiten $n^{\circ} 351$

Niemeyer, Tübingen : 153-162 


\title{
Aspect zéro et dépendance situationnelle: l'exemple du wolof
}

\author{
Stéphane ROBERT \\ (CNRS - LLACAN Paris)
}

\section{Introduction}

Le système verbal du wolof $^{1}$ comporte une conjugaison baptisée notamment Aspect zéro (Sauvageot, p.102) ${ }^{2}$ ou Aoriste (Robert 1995) ${ }^{3}$, qui présente des degrés de dépendance syntaxique variables ainsi que des valeurs sémantiques très diverses allant du gnomique à la consécution en passant par le passé historique, l'injonction ou d'autres formes de subordination. La malléabilité temporelle de cette forme qui peut renvoyer selon les cas à un passé, un présent ou un futur, et sa valeur aspectuelle ponctuelle semblent indiquer que l'Aoriste désigne la notion verbale hors de tout ancrage situationnel (Robert 1991, 199-234).

Ces caractérisques expliquent vraisemblablement la morphologie réduite de cette conjugaison qui peut prendre une forme zéro en présence d'un sujet lexical. Or il semble que l'on retrouve sous divers noms une telle forme verbale dans des langues variées ${ }^{4}$. En effet, avec des différences liées à l'organisation générale du système spécifique de chaque langue, ces diverses "formes nues" (ou

\footnotetext{
${ }^{1}$ Langue africaine du groupe ouest-atlantique parlée principalement au Sénégal.

2 Ou encore Minimal, Narratif ou Subordinatif. Pour une étude de l'ensemble du système verbal, une présentation beaucoup plus détaillée des emplois de l'Aoriste et des différentes analyses proposées, on pourra se reporter à Robert 1991.

${ }^{3}$ Le présent article reprend en effet, sous un angle différent, une analyse de cette conjugaison que j'ai proposée dans un article soumis en 1992 pour un volume d'hommage à Antoine Culioli et paru en 1995.

${ }^{4}$ Notamment le samo, le haoussa, l'arabe marocain (cf Robert 1995, 387-389).
} 
réduites) présentent une palette d'emplois comparable à celle de l'Aoriste du wolof.

Pour rendre compte de ces emplois dont la variété est problématique - mode du récit ou mode subordinatif -, on propose ici de définir une forme particulière de dépendance que l'on appellera dépendance situationnelle. Il s'agit d'un mode particulier d'ancrage dans le temps et de prise en charge assertive indirects par un repère extérieur. L'étude de l'Aoriste amène alors à expliciter certains mécanismes fondamentaux de l'assertion qui permettent de construire un énoncé complet, comme la nécessaire spécification du repère situationnel, mécanismes que l'on retrouve ailleurs notamment dans l'analyse des complétives, du subjonctif ou de formes verbales liées.

\section{Différents degrés de dépendance syntaxique}

L'Aoriste présente deux grandes catégories d'emplois apparemment contradictoires. Il apparaît en effet dans certaines propositions indépendantes tout en étant le mode par excellence de la subordination, obligatoire dans la plupart de subordonnées et suffisant à lui seul à construire un lien de subordination entre deux propositions lorsqu'il est employé en apodose. Le détail des emplois révèle en fait toute une palette dans les degrés de dépendance.

En indépendante, en effet, l'Aoriste est employé comme aoriste de récit dans les contes et récits historiques. Ce type d'emploi a longtemps été considéré comme le plus caractéristique. Mais ce mode est utilisé également dans les indications scéniques et les proverbes et, avec un degré de dépendance croissant, dans certaines exclamatives en réponse, dans des propositions injonctives et dans des interrogatives.

Par ailleurs, comme mode subordonnant, l'Aoriste est employé directement en apodose sans morphème subordonnant, dans des propositions à valeur finale ou consécutive ainsi que dans les complétives. Il est le seul mode à présenter de ce type d'emploi en asyndète. De plus, avec morphème subordonnant, l'Aoriste est obligatoire dans toutes les subordonnées, exception faite des causales, des complétives introduites par ne et des comparatives. 
En indépendante :

- aoriste de récit dans les contes et récits historiques

- dans les indications scéniques et les proverbes

- dans des propositions injonctives

- dans des interrogatives.

\section{Comme mode subordonnant :}

- sans morphème subordonnant:

- propositions à valeur finale ou consécutive

- complétives

- avec morphème subordonnant:

- l'aoriste est obligatoire dans toutes les subordonnées

sauf causales, complétives (en ne) et comparatives.

Cette diversité syntaxique apparemment contradictoire est d'autant plus problématique qu'elle s'accompagne d'effets sémantiques et de contraintes spécifiques qu'une simple indifférenciation temporelle ne saurait expliquer.

\section{Indépendance syntaxique et dépendance situationnelle}

Un examen détaillé montre que dans ses emplois les plus indépendants, l'Aoriste n'apparaît en réalité jamais en premier: il s'articule nécessairement à un élément précèdent qui spécifie le cadre situationnel à partir duquel va se dérouler le récit à l'Aoriste. Ainsi, dans les contes et récit historiques, l'Aoriste ne se présente jamais comme la première forme verbale. De manière caractéristique dans l'exemple suivant ${ }^{5}$, le récit s'ouvre sur une première référence temporelle marquée par un complément de temps, une forme verbale d'Emphatique du Verbe (dafa $a m$ ) et un suffixe de translation dans le passé (-oon). C'est seulement ensuite que le récit se déroule à l'Aoriste $(\mathrm{Mu} \text { woote... })^{6}$ :

5 cf Kesteloot et Mbodj (p.175). Les autres exemples sont tirés de la pièce de théâtre de Dieng Ba et Mbaye, du recueil de proverbes de Ndiaye Samb ou encore d'énoncés que j'ai recueillis en situation.

6 Les "conjugaisons" du wolof constituent en réalité un syntagme verbal en deux parties: un lexème verbal invariable et un morphème flexionnel qui lui est suffixé, postposé ou antéposé (comme dans le cas de l'Aoriste). 
Ca jamano yu yàgg, dafa amoon benn ilimaanu jàkka. Mu woote ca jàkka ja. Waa dëkk ba yépp wuyuji. Mu ne leen...

"En des temps lointains, il y avait (Emphatique du Verbe) un imam de mosquée. Il convoqua (Aoriste) une palabre à la mosquée. Tous les villageois s'y rendirent (Aoriste). Il leur dit (Aoriste)...."

L'opposition entre Parfait et Aoriste dans les emplois en récit (Robert 1991, 207-8) montre que les procès à l'Aoriste renvoient à une vérité "historique" parce qu'il n'indiquent par eux-mêmes ni spécifications temporelles, ni prise en charge assertive: leur vérité est indépendante de l'énonciateur. Mais les contraintes d'emploi montrent que cette rupture énonciative ne consiste pas en une simple indétermination, elle s'accompagne d'une dépendance de l'Aoriste par rapport au repère $^{7}$ temporel initial. Pour pouvoir enchaîner des événements qui ne sont pas construits par l'énonciateur depuis le moment de l'énonciation, il faut alors que l'Aoriste s'articule à une situation de référence spécifiée au préalable.

Cette particularité assertive est confirmée par les autres emplois "indépendants" de l'Aoriste. Dans les indications scéniques comme dans les proverbes, en effet, la proposition à l'Aoriste se présente comme une proposition syntaxiquement indépendante mais qui nécessite un ancrage situationnel extérieur dont elle dépend. On comprend alors qu'elle puisse prendre différentes valeurs temporelles en fonction de ce repère situationnel contextuel. Ainsi, dans le cas des indications scéniques, l'énoncé à l'Aoriste recevra sa spécification situationnelle à chaque représentation: il est donc validé dans ce présent à chaque fois recommencé.

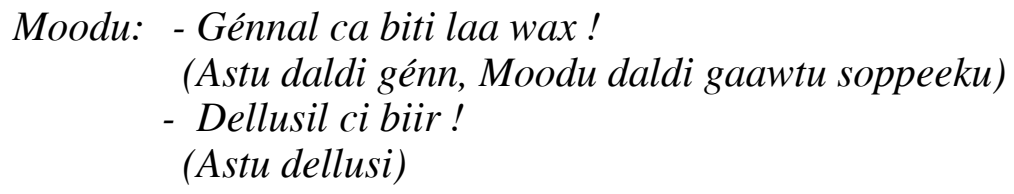

Moodu: - Génnal ca biti laa wax! (Astu daldi génn, Moodu daldi gaawtu soppeeku)

- Dellusil ci biir! (Astu dellusi)

Modou: - "Sors je t'ai dit !"

\footnotetext{
7 La notion de repère (ainsi que celle de rupture) est empruntée à Antoine Culioli. Pour Culioli, la valeur référentielle d'un énoncé est construite par toute une série de mises en relation intervenant aux niveaux notionnel, prédicatif et énonciatif. C'est cette opération fondamentale commune que Culioli appelle repérage. Voir notamment 1978 a et $1978 b$.
} 
(Astou sort aussitôt, Modou se change rapidement)

- "Reviens !"

(Astou revient)

C'est bien encore l'indépendance de l'Aoriste par rapport au temps, lieu et sujet de l'énonciation qui donne au proverbe sa valeur gnomique $^{8}$. L'énoncé est alors utilisé pour illustrer un contexte de discours qui lui sert de repère: le proverbe est donc vrai a priori en même temps qu'à chaque fois qu'on le prononce dans une situation donnée.

Ku yàgg dox, yàgg gis

qui (aoriste) $)^{9}$ durer marcher, (aoriste) durer voir

"Celui qui marche longtemps, voit beaucoup de choses"

(Le voyage donne de l'expérience)

Ainsi, quoique syntaxiquement indépendant, l'énoncé à l'Aoriste ne comportant par lui-même ni spécifications temporelles ni prise en charge par l'énonciateur, dépend de l'élément extérieur qui lui sert de repère situationnel. Faute de cet ancrage temporel et assertif préalable, la proposition à l'Aoriste, en attente de spécifications situationnelles, sera interprétée comme une interrogation ou une injonction, c'est-à-dire comme une demande de prise en charge: l'absence de repère contextuel entraîne un recours à autrui.

L'Aoriste est en effet obligatoire avec les morphèmes interrogatifs en $-u$ qui indiquent une absence de détermination spatiale:

\section{Lu $\tilde{n} u$ wax?}

8 On notera au passage les liens entre détermination nominale et valeur aspectomodale de l'énoncé: les proverbes à l'Aoriste s'ouvrent en effet sur un relatif indéterminé $(k u)$ qui permet un décrochage par rapport à la situation d'énonciation et confère à l'énoncé une interprétation générique. Ils s'opposent en cela aux exclamatives intensives à l'Aoriste qui sont construites avec un relatif démonstratif (Robert 1991, 209-211). Déchaine (1991, 36-38) relève un rôle comparable de la détermination nominale dans l'interprétation sémantique d'un temps "indéfini" à propos du haïtien et de l'anglais.

${ }^{9}$ La marque d'aoriste antéposée au lexème verbal est ici sous la forme zéro qui peut apparaître à la 3ème personne. 
quoi ils+Aor dire?

"Qu'ont-ils dit?"

Il peut apparaître également en reprise, sans morphème interrogatif, comme une demande de confirmation, mi-exclamative, mi-interrogative. Reprenant un énoncé déjà asserté, l'énonciateur remet alors en cause la proposition qui avec l'Aoriste n'est pas prise en charge par lui-même. D'où les effets de réorientation argumentative par une mise en doute: Kon ma delloo la xaalis bi ? "(Il faut) donc que je te rende l'argent ?" (Robert 1991, 211-212)10.

En valeur injonctive enfin, l'Aoriste ne s'articule à aucun énoncé qui lui serve de repère assertif ${ }^{11}$ :

[on frappe]

Mu duggsi !

il+Aor entrer+allatif

"(qu') il entre !"

\section{Subordination et intégration syntaxique du repérage situationnel}

Les contraintes sur les emplois indépendants de l'Aoriste ont montré que l'absence de repérage énonciatif entraînait une dépendance par rapport à un élément contextuel extérieur apportant à cette conjugaison l'ancrage situationnel qui lui fait défaut. Cette dépendance situationnelle fait de l'Aoriste le mode privilégié des propositions qui développent les éléments d'une situation indiquée par une proposition précédente. On comprend alors qu'il soit obligatoire dans les subordonnées de temps, de lieu, de manière ou encore les

10 On trouve encore d'autres exclamatives à l'Aoriste présentant une valeur d'intensif (ibid.: 209-211). Celles-ci reposent sur le même mécanisme assertif mais dans des enchaînements discursifs différents.

11 Il ne peut cependant apparaître directement et s'enchaîne soit à un contexte énonciatif qui construit une incertitude ou une attente (voir ci-dessous), soit à un Impératif (cf Robert 1991, 213-4). Ce dernier fonctionne comme repère temporel car il est déterminé par rapport au moment de l'énonciation (ordre valable au moment de l'énonciation) mais pas comme repère assertif (recours à autrui). Voir Robert (1991, 235-256). 
relatives qui développent les actants de la principale, ceci avec les valeurs temporelles spécifiées par le contexte.

Defal lii ma la wax

faire+Impér.sg ce-que je+Aor te dire

"Fais ce que je t'ai dit"

Fii nu tollu tey, jébbal naa la sama bopp...

Ici nous+Aor arriver-à aujourd'hui, remettre je+Pft te ma tête ...

"Au point où on en est aujourd'hui, je m'en remets à toi..."

Dinaa ko ko wax bu ñówee

je+Futur lui le dire quand (Aor) venir+antériorité

"Je le lui dirai, quand il viendra"

Bu ñówaan xale yépp dañuy bég

Quand (Aor) venir+répét.passé, enfant tous ils+EmphVb+inacc être-satisfait

"Quand il venait, tous les enfants étaient contents"

La rupture par rapport à la situation d'énonciation marquée par l'Aoriste explique qu'il soit également le mode des hypothétiques:

Boo jëndul, bàyyil

si+tu+Aor acheter+nég, laisser+Impér.sg

"Si tu n'achètes pas, laisse tomber"

En revanche, l'Aoriste qui place la proposition dans l'orbite situationnelle et assertive de ce qui précède est impossible dans les subordonnées qui indiquent une prise en charge de l'énonciateur comme les causales.

Dans tous les cas qui précèdent, la proposition à l'Aoriste était introduite par un morphème subordonnant. Mais cette conjugaison est aussi le mode privilégié de la parataxe. Employé en apodose, il est en effet le seul mode qui construit un lien de subordination entre deux propositions sans aucun morphème subordonnant ${ }^{12}$. On affaire alors aux cas où ce mode semble construire un lien de dépendance maximal. Celui-ci s'explique par le statut syntaxique de l'élément qui

12 Un enchaînement au Parfait indiquera une successivité temporelle. Les autres modes sont impossibles en apodose. Pour plus de détails voir Robert 1991 (217$220)$ et 1995 (381-382). 
lui sert de repère. Les emplois de l'Aoriste en parataxe se ramènent en effet à deux grands cas, quel que soit le mode de la protase: ceux où la proposition à l'Aoriste a valeur consécutive ou finale et ceux où elle est en fonction complétive. Ces deux cas manifestent une même dépendance situationnelle mais correspondent à un lien syntaxique différent de la proposition à l'Aoriste avec l'élément qui lui sert de repère situationnel.

Dans le cas de la complétive, la proposition à l'Aoriste est sujet ou objet d'un verbe qui lui sert de repère situationnel. On a donc un double lien entre les deux propositions: la proposition à l'Aoriste remplit une place argumentale dans la relation prédicative qui précède (ou qui suit s'il s'agit d'une complétive sujet), cette dernière en revanche apporte les spécifications temporelles et assertives qui manquent à la proposition à l'Aoriste. La dépendance est dissymétrique mais double.

\section{Damaa bëgg mu xam kã̃ laa ñëw}

je+EmphVb vouloir il+Aor. savoir quand je+EmphComp venir

"Je veux (qu)'il sache quand je suis venu"

Dans le cas de la valeur consécutive ou finale, c'est toute la proposition précédente qui sert de repère situationnel à la proposition à l'Aoriste. Cette dernière dépend en effet d'un ancrage situationnel préalable mais, à l'inverse, il suffit que celui-ci soit spécifié pour qu'elle soit validée. D'où le lien de consécution automatique ${ }^{13}$ produit par l'enchaînement des deux propositions. La première entraîne la seconde. La proposition à l'Aoriste prend alors une valeur consécutive si la proposition repère renvoie à un procès déjà accompli, et une valeur finale s'il est seulement visé ou inaccompli.

\section{Dafa toog ci siis bi, mu dàmm}

il+EmphVb s'asseoir dans chaise la, elle+Aor se-casser

"Il s'est (à peine) assis sur la chaise (qu)'elle s'est cassée"

\footnotetext{
13 Sous une autre forme, on retrouve ce même effet de consécution dans l'enchaînement des récits historiques à l'Aoriste: à partir du premier repère, les événements semblent se dérouler de manière inéluctable car ils s'enchaînent entre eux, indépendamment de l'énonciateur. Les finales ou consécutives sont l'équivalent, en subordonnée de ce qui dans le récit apparaît comme le déterminisme de l'histoire.
} 
Tàll nañu lamp ya, mu leer nañ̃ foofa

allumer ils+Pft lampes les+éloignés, il+Aor être-lumineux très-lumineux làbas

"On a allumé des lampes (si bien que) il y a une clarté éblouissante là-bas"

Maay doktoor biy gëdd feebar mu jóge ci nit ki

je+EmphSujet+inacc docteur qui+inacc gronder maladie elle+Aor partir dans homme le

"Je suis le docteur qui gronde la maladie (pour qu') elle quitte la personne"

Les emplois subordonnants de l'Aoriste correspondent donc à une intégration syntaxique du repère situationnel: la proposition à l'Aoriste a pour repère situationnel un élément extérieur avec lequel elle entretient par ailleurs une relation syntaxique de dépendance.

\section{Le repère situationnel comme variable contextuelle}

La proposition à l'Aoriste a donc toutes les déterminations d'un énoncé complet mais il lui manque la prise en charge assertive et les spécifications temporelles, c'est-à-dire son repère énonciatif. Le procès est posé par rapport à une situation non spécifiée, ce qui explique ses compatibilités. La proposition dépend alors de l'élément extérieur qui va spécifier ce repère en lui fournissant une situation de référence. Cette dépendance qu'on appellera situationnelle est définitoire. Elle se manifeste dans l'attente de prise en charge assertive que l'on relève lorsque le repère n'a pas été spécifié dans le contexte précédent. Elle explique également le lien nécessaire et suffisant entre la proposition à l'Aoriste et son repère pour constituer un énoncé complet et validé.

A partir de cette description et en faisant varier la nature syntaxique de l'élément qui sert de repère situationnel à la proposition à l'Aoriste, on peut expliquer les différents emplois de celle-ci, les différents degrés de dépendance qu'ils manifestent et les valeurs sémantiques qui leur sont attachées.

Le procès est prédiqué dans une situation non spécifiée :

- Absence de déterminations énonciatives 
(prise en charge assertive et spécifications temporelles)

- Dépendance par rapport à un repère situationnel extérieur

- Lien nécessaire et suffisant entre la proposition à l'Aoriste et son repère pour constituer un énoncé complet et validé.

\begin{tabular}{|c|l|l|}
\hline Repère situationnel & \multicolumn{1}{|c|}{ Valeur } & \multicolumn{1}{|c|}{ Emplois } \\
\hline une situation passée & Rupture & - contes, récits historiques \\
\hline une situation actuelle & $\begin{array}{l}\text { Illustration de la situation } \\
\text { d'énonciation }\end{array}$ & $\begin{array}{l}\text { - indications scéniques } \\
\text { - proverbes }\end{array}$ \\
\hline Zéro & Recours à autrui & $\begin{array}{l}\text { - Injonction } \\
\text { - Interrogation }\end{array}$ \\
\hline $\begin{array}{l}\text { Une autre proposition } \\
\text { Un verbe transitif }\end{array}$ & $\begin{array}{l}\text { Dépendance syntaxique } \\
\text { Dépendance syntaxique }\end{array}$ & $\begin{array}{l}\text { - consécutive / finale } \\
\text { - complétive }\end{array}$ \\
\hline
\end{tabular}

L'élément repère apparaît donc ici comme la variable contextuelle qui permet d'appréhender les emplois très divers d'un même morphème sous la forme d'un mécanisme régulier de spécification contextuelle.

\section{Conclusion : noyau prédicatif et noyau assertif}

Le fonctionnement particulier de cette conjugaison montre qu'une relation prédicative doit être définie par rapport à une situation de référence pour constituer un énoncé complet. Il prouve également que la situation cardinale de référence est la situation d'énonciation définie par ses deux paramètres: le moment de l'énonciation, origine des déterminations temporelles, et le sujet de l'énonciation, origine de la prise en charge assertive. En effet, l'absence de spécifications par rapport à la situation d'énonciation marquée par l'Aoriste ne fonctionne pas comme une simple indéfinition mais définit en outre 
une attente de déterminations qui se manifeste par une dépendance structurelle.

On voit apparaître là un mécanisme fondamental de l'assertion: l'absence de prise en charge assertive entraîne une sorte d'anaphore situationnelle dans la mesure où, à défaut de déterminations par rapport au moment de l'énonciation, c'est du contexte précédent que l'énoncé tire sa référence situationnelle. L'Aoriste entretient donc avec son repère situationnel une relation comparable à celle d'un pronom avec son antécédent. En l'absence d'antécédent, le relatif comme l'Aoriste prendra une valeur interrogative. On peut donc dire que l'ancrage situationnel constitue ici une place vide non instanciée ${ }^{14}$. La notion de dépendance situationnelle exprime ce rapport anaphorique entre la proposition en attente de déterminations situationnelles et l'élément qui spécifie le repère situationnel.

Cette analyse devrait permettre de reformuler de manière systématique, les problèmes de portée de l'assertion soulevés par la subordination et les liens entre dépendance énonciative et dépendance syntaxique qui s'y manifestent. On entrevoit comment opposer au moins deux types d'énoncés complexes: ceux dans lesquels il y a deux noyaux prédicatifs mais un seul noyau assertif au niveau de la principale, la subordonnée étant alors dans l'orbite assertive de la protase; et ceux où, aux deux noyaux prédicatifs, correspondent deux noyaux assertifs:

proposition $_{1}$ (repérage énonciatif), proposition 2 (anaphore situationnelle)

proposition1 (repérage énonciatif), proposition 2 (repérage énonciatif)

Ces deux cas correspondent notamment à l'opposition entre relative à l'indicatif et relative au subjonctif en français. Dans je cherche une maison qui soit sur la colline, l'existence de la maison n'est posée que

\footnotetext{
14 Pour plus de détails, voir Robert (1991, 225-230). Cette analyse est convergente avec celle que pose Rose-Marie Déchaine (1991, 31-50) en termes générativistes pour les "phrases à formes nues" (bare sentences). L'auteur refuse en effet de poser un opérateur temporel caché ou une absence d"'inflexion" et préfère considérer qu'il y a une "Inflexion projection with no inherent features". Ce phénomène reçoit ici une explication plus générale grâce à la théorie de l'énonciation qui pose un repèrage énonciatif nécessaire à tout énoncé.
} 
par rapport à la recherche que j'en fais, alors que dans je cherche une maison qui est sur la colline, le sujet est à la recherche d'une maison qui existe bel et bien indépendamment de sa recherche.

On opposera ainsi également les deux types de complétives du wolof : celles à l'Aoriste qui ne comportent pas d'ancrage énonciatif propre (dépendance temporelle et assertive), et celles en ne+ Parfait qui comportent un ancrage à la fois temporel et assertif indépendant de celui de la principale ${ }^{15}$ :

Yaakaar naa nga tane

Espérer je+Pft tu+Aor. aller-

mieux

"J'espère que tu $\underline{\text { iras }}$ mieux" (Aoriste)

Yaakaar naa ne tane nga

Espérer je+Pft que aller-mieux $\mathrm{tu}+\mathrm{Pft}$

"J'espère que tu vas mieux

"(Parfait)

L'existence des deux paramètres (temps et sujet) dans le repérage énonciatif permet de rendre compte des effets à la fois modaux et temporels de l'absence d'ancrage énonciatif dans la subordonnée.

On signalera enfin le double fonctionnement de la conjonction ndax en wolof. Suivant le mode employé dans la subordonnée (Aoriste ou Emphatique du Verbe) et le repérage qu'il marque (dépendance ou repérage énonciatif propre), on relève un remarquable changement de sens de la proposition:

15 Le grec moderne présente lui aussi une opposition assertive comparable entre complétives en oti+ indicatif et complétives au subjonctif (en $n a$ ), ce dernier mode prenant par ailleurs des valeurs de visée ou de conation (voir Delveroudi et alii, 185199). 


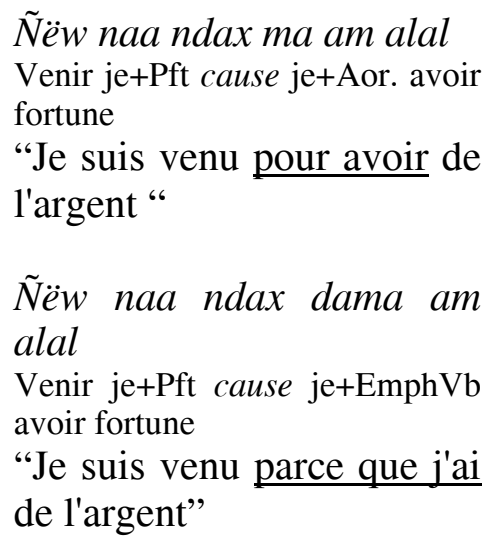

En faisant jouer la dépendance situationnelle sur l'un, l'autre ou les deux paramètres énonciatifs, on voit s'ouvrir la perspective d'une distinction encore plus fine entre les différents cas de dépendance: dépendance assertive mais repérage temporel propre ou dépendance assertive et dépendance temporelle.

\section{Bibliographie}

Culioli, Antoine (1978a): "Valeurs modales et opérations énonciatives". - In: Le Français moderne 46 (4), 300-317.

- (1978b, 1983): "Valeurs aspectuelles et opérations énonciatives: la notion d'aoristique". - In: S.Fisher, J.J. Franckel (eds): Enonciation: aspect et détermination (Paris, EHESS), 99-114.

Déchaine, Rose-Marie (1991): "Bare Sentences". - In: S. Moore, A. Wyner (eds): SALT I, Proceedings of the First Semantics and Linguistic Theory Conference. - (= Cornell University Working Papers in Linguistics 10), 31-50.

Delveroudi, Rhéa, I. Tsamadou, S. Vassilaki (1994): "Mood and Modality in Modern Greek: the particle NA". - In: I. PhilippakiWarburton (ed): Themes in Greek Linguistics. (AmsterdamPhiladelphia John Benjamins) (= Current Issues in Linguistic Theory 117), 185-199.

Dieng Ba, Assy, C. Mbaye (s.d.): Xët cig lëndëm: adaptation de L'ombre et la lumière de Salif Dione. - Dakar: manuscrit de travail de la troupe du théâtre Daniel Sorano de Dakar.

Kesteloot, Lilyan, C. Mbodj (1983): Contes et mythes wolof. - Dakar: Nouvelles Editions Africaines. 
Ndiaye Samb, El-Hadj Amadou (s.d.): Sagesse africaine (Proverbes wolof). - Dakar: offset.

Robert, Stéphane (1991): Une approche énonciative du système verbal : le cas du wolof. - Paris: Editions du CNRS.

- (1995): "Aoristique et mode subordinatif: liens entre aspect et prédication". - In: J.J. Franckel, J. Bouscaren, S. Robert (sous la direction de): Langues et langage: problèmes et raisonnement en linguistique (Mélanges offerts à Antoine Culioli), (Paris: P.U.F), 373389.

Sauvageot, Serge (1965): Description synchronique d'un dialecte wolof: le parler du Dyolof. - Dakar, IFAN. 